\title{
Small-molecule inhibition of proteasome and aggresome function induces synergistic antitumor activity in multiple myeloma
}

\section{Citation}

Hideshima, T., J. E. Bradner, J. Wong, D. Chauhan, P. Richardson, S. L. Schreiber, and K. C. Anderson. 2005. "Small-Molecule Inhibition of Proteasome and Aggresome Function Induces Synergistic Antitumor Activity in Multiple Myeloma." Proceedings of the National Academy of Sciences 102 (24): 8567-72. https://doi.org/10.1073/pnas.0503221102.

\section{Permanent link}

http://nrs.harvard.edu/urn-3:HUL.InstRepos:41467492

\section{Terms of Use}

This article was downloaded from Harvard University's DASH repository, and is made available under the terms and conditions applicable to Other Posted Material, as set forth at http:// nrs.harvard.edu/urn-3:HUL.InstRepos:dash.current.terms-of-use\#LAA

\section{Share Your Story}

The Harvard community has made this article openly available.

Please share how this access benefits you. Submit a story.

\section{Accessibility}




\title{
Small-molecule inhibition of proteasome and aggresome function induces synergistic antitumor activity in multiple myeloma
}

\author{
Teru Hideshima*, James E. Bradner*†, Jason Wong ${ }^{\dagger}$, Dharminder Chauhan*, Paul Richardson*, Stuart L. Schreiber ${ }^{\dagger \ddagger \S}$, \\ and Kenneth C. Anderson*§

\begin{abstract}
*Jerome Lipper Multiple Myeloma Center, Department of Medical Oncology, Dana-Farber Cancer Institute, Harvard Medical School, 44 Binney Street, Boston, MA 02115; ' Broad Institute of Harvard University and Massachusetts Institute of Technology, 320 Charles Street, Cambridge, MA 02141; and ${ }^{\ddagger}$ Howard Hughes Medical Institute, Department of Chemistry and Chemical Biology, Harvard University, 12 Oxford Street, Cambridge, MA 02138
\end{abstract} \\ Contributed by Stuart L. Schreiber, April 20, 2005
}

We have shown that the proteasome inhibitor bortezomib (formerly known as PS-341) triggers significant antitumor activity in multiple myeloma (MM) in both preclinical models and patients with relapsed refractory disease. Recent studies have shown that unfolded and misfolded ubiquitinated proteins are degraded not only by proteasomes, but also by aggresomes, dependent on histone deacetylase 6 (HDAC6) activity. We therefore hypothesized that inhibition of both mechanisms of protein catabolism could induce accumulation of ubiquitinated proteins followed by significant cell stress and cytotoxicity in MM cells. To prove this hypothesis, we used bortezomib and tubacin to inhibit the proteasome and HDAC6, respectively. Tubacin specifically triggers acetylation of $\alpha$-tubulin as a result of HDAC6 inhibition in a dose- and time-dependent fashion. It induces cytotoxicity in MM cells at $72 \mathrm{~h}$ with an IC $\mathrm{C}_{50}$ of $5-20 \mu \mathrm{M}$, which is mediated by caspase-dependent apoptosis; no toxicity is observed in normal peripheral blood mononuclear cells. Tubacin inhibits the interaction of HDAC6 with dynein and induces marked accumulation of ubiquitinated proteins. It synergistically augments bortezomib-induced cytotoxicity by c-Jun $\mathrm{NH}_{2}$-terminal kinase/caspase activation. Importantly, this combination also induces significant cytotoxicity in plasma cells isolated from MM patient bone marrow. Finally, adherence of MM cells to bone marrow stromal cells confers growth and resistance to conventional treatments; in contrast, the combination of tubacin and bortezomib triggers toxicity even in adherent MM cells. Our studies therefore demonstrate that tubacin combined with bortezomib mediates significant anti-MM activity, providing the framework for clinical evaluation of combined therapy to improve patient outcome in MM.

histone deacetylase

M ultiple myeloma (MM) is a plasma cell malignancy that remains incurable despite conventional treatment (1) or high-dose therapy and stem cell transplantation (2). Novel agents have recently been developed that target not only MM cells, but also the bone marrow (BM) microenvironment, and can overcome conventional drug resistance (3). For example, the proteasome inhibitor bortezomib (formally PS-341) induces significant antitumor activity in human MM cell lines and freshly isolated patient MM cells (3-11) associated with c-Jun $\mathrm{NH}_{2-}$ terminal kinase (JNK) (also known as stress-activated protein kinase) and caspase activation, followed by apoptosis $(4,5,9)$. Bortezomib also inhibits adherence of MM cells to BM stromal cells (BMSCs) by down-regulating adhesion molecules (intercellular adhesion molecule-1 and vascular cell adhesion molecule 1) (12) and induces cleavage of DNA-protein kinase catalytic subunit, suggesting that bortezomib also inhibits DNA repair. Neither IL-6 nor adherence of MM cells to BMSCs protects against bortezomib-induced apoptosis. These data further support our observation that bortezomib enhances sensitivity and can overcome resistance in MM cells to conventional chemo- therapeutic agents, especially to DNA-damaging agents (7). Importantly, a phase II trial of bortezomib treatment of 202 patients with refractory relapsed MM demonstrated 35\% responses, including $10 \%$ complete and near-complete responses (13); however, $65 \%$ of patients did not respond. However, resistance to bortezomib, to date, is not fully understood. We have recently demonstrated that heat shock protein (hsp)-27 mediates bortezomib resistance; conversely, inhibiting hsp-27 expression by using hsp-27 antisense, p38 mitogen-activated protein kinase (MAPK) small interfering RNA (siRNA), or p38 MAPK inhibitor to down-regulate hsp-27 can restore MM cell susceptibility to bortezomib $(8,11)$.

Recent studies have revealed an alternative system to the proteasome for degradation of polyubiquitinated misfolded/ unfolded proteins, termed the aggresome $(14,15)$. Aggresome formation ultimately induces autophagic clearance, which terminates in lysosomal degradation. The aggresome pathway therefore likely provides a novel system for delivery of aggregated proteins from cytoplasm to lysosomes for degradation (16). In aggresomal protein degradation, histone deacetylase 6 (HDAC6) has an essential role because it can bind both polyubiquitinated proteins and dynein motors, thereby acting to recruit protein cargo to dynein motors for transport to aggresomes (17). In the present study, we hypothesized that inhibition of both proteasomal and aggresomal protein degradation systems could induce accumulation of polyubiquitinated proteins and significant cell stress, followed by activation of apoptotic cascades. In this context, we used bortezomib to inhibit the proteasome and tubacin, which specifically inhibits HDAC6 $(18-20)$ to block the aggresome. We show that tubacin combined with bortezomib induces synergistic cytotoxicity in MM cell lines and freshly isolated BM plasma cells (BMPCs) from MM patients, providing the preclinical rationale for clinical protocols of this combination to improve patient outcome in MM.

\section{Materials and Methods}

Cell Lines, Patient BMPCs, and BMSCs. Dexamethasone-sensitive (MM.1S) and -resistant (MM.1R) human MM cell lines were kindly provided by Steven Rosen (Northwestern University, Chicago). RPMI8226 and U266 human MM cell lines were obtained from the American Type Culture Collection. The IL-6-dependent INA-6 cell line was kindly provided by Martin Gramatzki (University of Erlangen-Nuernberg, Erlangen, Ger-

Abbreviations: HDAC, histone deacetylase; $\mathrm{MM}$, multiple myeloma; $\mathrm{BM}$, bone marrow BMSC, BM stromal cell; BMPC, BM plasma cell; JNK, c-Jun $\mathrm{NH}_{2}$-terminal kinase; hsp, heat shock protein; siRNA, small interfering RNA; MTT, 3-(4,5-dimethylthiazol-2-yl)-2,5-diphenyl tetrazolium bromide; Ub, ubiquitin; PARP, poly(ADP-ribose) polymerase; SAHA, suberoylanilide hydroxamic acid; PBMC, peripheral blood mononuclear cell.

§To whom correspondence may be addressed. E-mail: stuart_schreiber@harvard.edu or kenneth_anderson@dfci.harvard.edu.

() 2005 by The National Academy of Sciences of the USA 

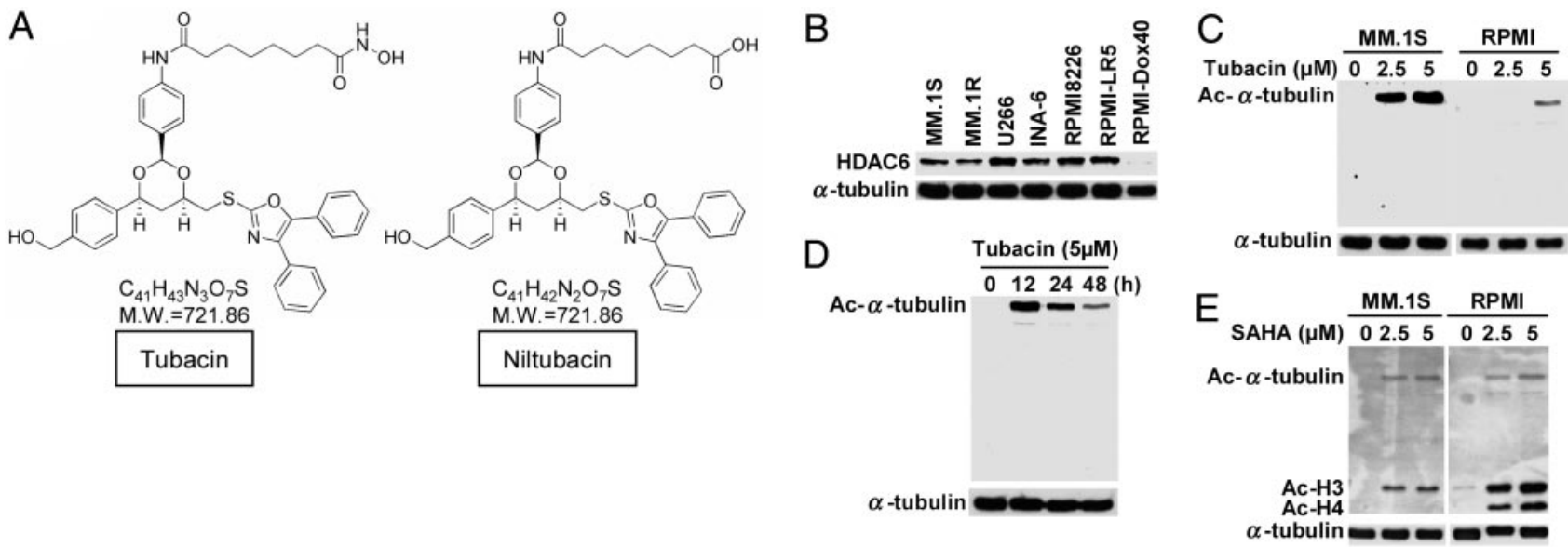

Fig. 1. Tubacin specifically induces acetylation of $\alpha$-tubulin in MM cells. (A) Chemical structures of tubacin and an inactive analog niltubacin. (B) Western blot of baseline expression of HDAC6 in MM cell lines. (C) MM.1S and RPMI8226 cells were cultured for $24 \mathrm{~h}$ in the presence ( 2.5 and $5 \mu \mathrm{M})$ or absence of tubacin. (D) RPMI8226 cells were cultured for the indicated times in the presence of tubacin $(5 \mu \mathrm{M})$. Whole-cell lysates were subjected to Western blot using anti-Ac lysine $\mathrm{Ab}$. Immunoblotting with anti- $\alpha$-tubulin serves confirms equal protein loading. (E) MM.1S and RPMI8226 cells were cultured for $24 \mathrm{~h}$ in the presence ( 2.5 and $5 \mu \mathrm{M})$ or absence of SAHA. Whole-cell lysates were subjected to Western blotting using anti-Ac lysine Ab. In contrast to tubacin, SAHA markedly triggers acetylation of histones $\mathrm{H} 3$ and $\mathrm{H} 4$.

many). Melphalan-resistant RPMI-LR5 and doxorubicinresistant RPMI-Dox40 cell lines were provided by William Dalton (H. Lee Moffitt Cancer Center, Tampa, FL). All MM cell lines were cultured in RPMI medium 1640 containing $10 \%$ FBS (Sigma), $2 \mu \mathrm{M}$ L-glutamine, 100 units $/ \mathrm{ml}$ penicillin, and 100 $\mu \mathrm{g} / \mathrm{ml}$ streptomycin (GIBCO). INA-6 cells were maintained with addition of IL-6 $(1 \mathrm{ng} / \mathrm{ml})$. MM patient plasma cells were purified from BM aspirates by negative selection by using an antibody mixture (RosetteSep Separation System, StemCell Technologies, Vancouver) as described (9). The purity of MM cells was $>90 \%$, as confirmed by flow cytometric analysis using anti-CD138 Ab (Pharmingen). Mononuclear cells separated by Ficoll-Hipaque density sedimentation from BM aspirates were also used to establish long-term BMSCs as described $(6,21)$. All experiments with patient samples were performed according to the protocol approved by the Institutional Review Board.
Reagents. A peptide boronate proteasome inhibitor bortezomib was provided by Millennium Pharmaceuticals (Cambridge, MA). HDAC6-specific inhibitor tubacin and its nonactive derivative niltubacin were obtained from the Broad Institute and the Massachusetts Institute of Technology (18).

Growth Inhibition Assay. The inhibitory effect of bortezomib and/or tubacin on MM cell growth was assessed by measuring 3-(4,5-dimethylthiazol-2-yl)-2,5-diphenyl tetrazolium bromide (MTT) dye absorbance as described (11). All experiments were performed in quadruplicate.

Immunoblotting. Cells cultured with tubacin and/or bortezomib were harvested, washed, and lysed by using lysis buffer $(50 \mathrm{mM}$, Tris $\cdot \mathrm{HCl}, \mathrm{pH} 7.4 / 150 \mathrm{mM} \mathrm{NaCl} / 1 \%$ Nonidet $\mathrm{P}-40 / 5 \mathrm{mM}$ EDTA/5 mM NaF/1 mM Na $3 V_{4} / 1 \mathrm{mM} \mathrm{PMSF} / 5 \mu \mathrm{g} / \mathrm{ml} \mathrm{leu-}$
A

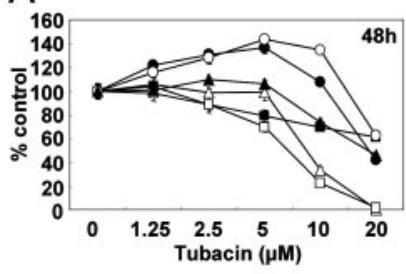

B

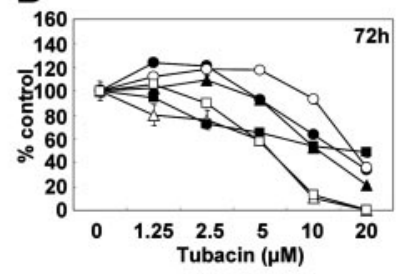

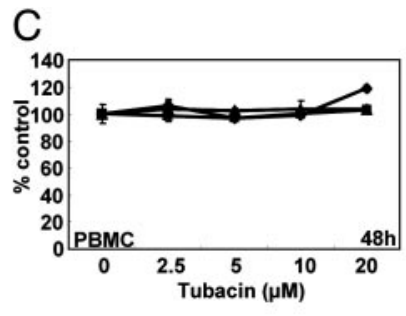
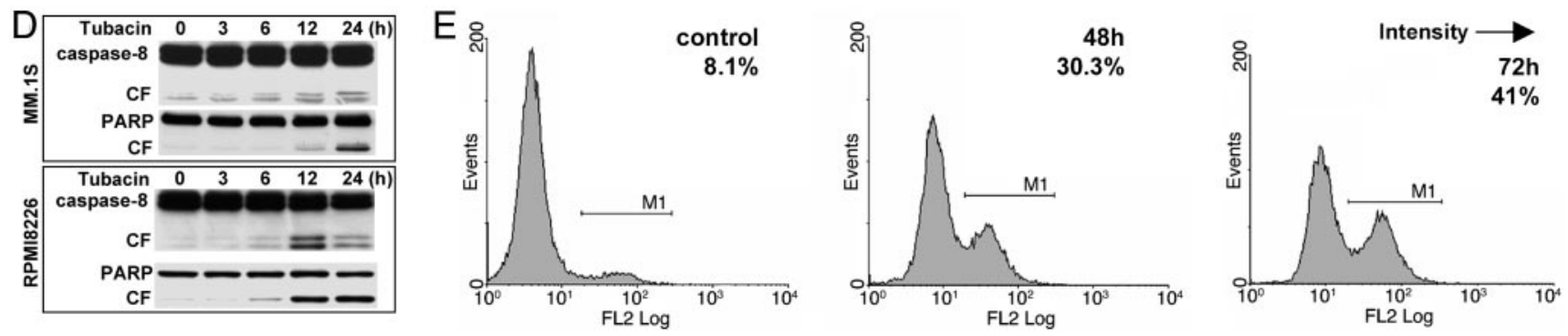

Fig. 2. Tubacin induces apoptosis by activation of caspases. ( $A$ and $B$ ) MM.1S ( $\bullet$ ), MM.1R (O), U266 ( $\Delta$ ), RPMI8226 ( $\triangle$ ), RPMI-LR5 ( $\square$ ), and RPMI-Dox40 ( $\square$ ) cells

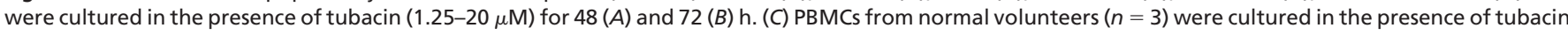
$(2.5-20 \mu \mathrm{M})$ for $48 \mathrm{~h}$. Cell growth was assessed by MTT assay, and data represent mean ( \pm SD) of quadruplicate cultures. (D) MM.1S and RPMI8226 cells were

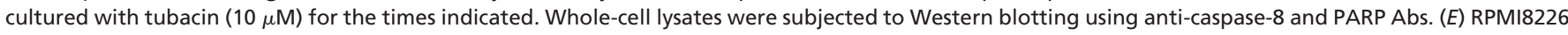
cells were cultured with tubacin $(10 \mu \mathrm{M})$ for 48 and $72 \mathrm{~h}$. Cells were subjected to APO2.7 staining to assess apoptosis by using flow cytometry. 
peptine $/ 5 \mu \mathrm{g} / \mathrm{ml}$ aprotinin). Whole-cell lysates were subjected to SDS/PAGE, transferred to nitrocellulose membrane (Bio-Rad), and immunoblotted with specific Abs (9). Western blotting was done by using anti-HDAC6, HDAC1, acetylated lysine, acetylated histone $\mathrm{H} 3$, acetylated histone $\mathrm{H} 4$, ubiquitin (Ub), phospho-stress-activated protein kinase, caspase-8, caspase-9, caspase-3, and poly(ADP-ribose) polymerase (PARP) Abs (Cell Signaling, Beverly, MA) with anti- $\alpha$-tubulin Ab (Santa Cruz Biotechnology) and anti-dynein Ab (Sigma). For immunoprecipitation, whole-cell lysates were incubated with anti-Ub or dynein Abs overnight at $4^{\circ} \mathrm{C}$, and then incubated with protein A/G PLUS-Agarose (Santa Cruz Biotechnology) for $2 \mathrm{~h}$ at $4^{\circ} \mathrm{C}$, as in our prior study (9). Immunoprecipitates were then subjected to Western blotting for detection of HDAC6, HDAC1, and dynein.

Flow Cytometric Analysis. For cell cycle analysis, MM.1S cells cultured for $24 \mathrm{~h}$ with tubacin and/or bortezomib were harvested, washed with PBS, fixed with $70 \%$ ethanol, and treated with $10 \mu \mathrm{g} / \mathrm{ml}$ of RNase (Roche Diagnostics). Cells were then stained with propidium iodine (Sigma) $(5 \mu \mathrm{g} / \mathrm{ml})$, and cell cycle profile was determined by using $\mathrm{M}$ software on an Epics flow cytometer (Coulter) (22).

Apoptotic cell death was assessed by staining with APO 2.7 Ab coupled to phycoerythrin (Immunotech, Marseille, France) as described (23).

Transient Transfection of HDAC6 siRNA. MM.1S cells were transiently transfected with HDAC6 siRNA (Dharmacon, Lafayette, CO) by using Cell Line Nucleofecto Kit V, according to the manufacturer's (Amaxa Biosystems, Gaithersburg, MD) instructions (11). After transfection, MM.1S cells were subjected to Western blotting and MTT assay in the presence or absence of bortezomib.

Growth of MM Cells Adherent to BMSCs. To evaluate the effect of combined tubacin and bortezomib treatment on growth of MM cells adherent to BMSCs, MM.1S and RPMI8226 cells were cultured for $24 \mathrm{~h}$ in BMSC-coated 96-well plates, in the presence or absence of tubacin and/or bortezomib. After treatment, DNA synthesis was measured by $\left[{ }^{3} \mathrm{H}\right]$ thymidine (PerkinElmer) uptake as described (22). All experiments were performed in quadruplicate.

Statistical Analysis. Statistical significance of differences observed in drug-treated versus control cultures was determined by using the Wilcoxon signed-ranks test. The minimal level of significance was $P<0.05$. The interaction between tubacin and bortezomib was analyzed by isobologram analysis using the CALCUSYN software program (Biosoft, Ferguson, MO) to determine whether the combination was additive or synergistic; a combination index $<0.7$ indicates a synergistic effect as described (24).

\section{Results and Discussion}

Tubacin Specifically Induces Acetylation of $\alpha$-Tubulin in MM Cell Lines. We first examined the baseline expression of HDAC6 in several MM cell lines. Although MM.1S, U266, INA-6, RPMI8226, and RPMI-LR5 MM cell lines constitutively express HDAC6, only low levels of HDAC6 are evident in RPMI-Dox-40 cells (Fig. 1A). Because tubacin induces acetylation of $\alpha$-tubulin in the A549 human lung cancer cell line by specific inhibition of HDAC6 activity (18), we next examined the effect of tubacin on acetylation of $\alpha$-tubulin in MM.1S and RPMI8226 MM cells. As shown in Fig. 1B, tubacin significantly induces acetylation of $\alpha$-tubulin in a dosedependent fashion in both MM.1S and RPMI8226 cells, without alteration of protein expression; importantly, no other acetylated proteins were recognized by Western blotting. Similar results were observed in INA-6 and RPMI-Dox40 cells (data not shown). We next assessed the dose-dependent effect of tubacin and show that
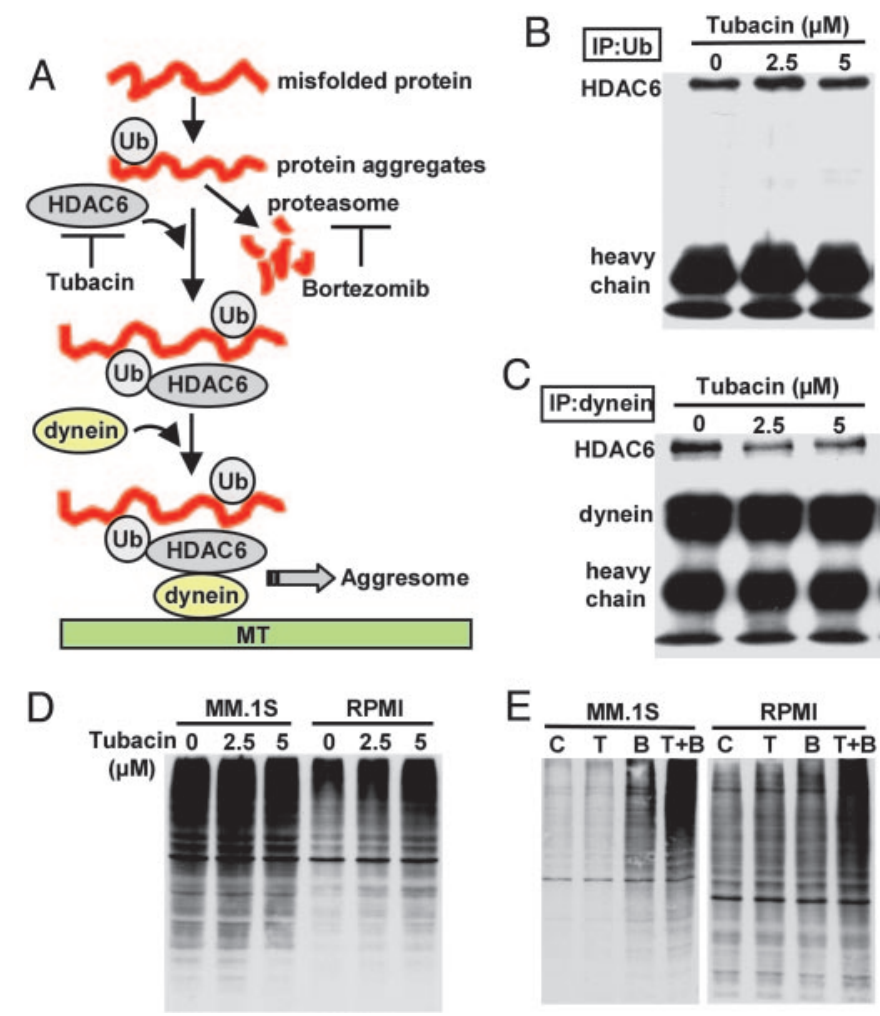

Fig. 3. Tubacin inhibits binding of HDAC6 with dynein, and when combined with bortezomib, it induces significant accumulation of polyubiquitinated proteins. $(A)$ Hypothetical rationale whereby tubacin enhances cytotoxicity induced by bortezomib [adapted from Kawaguchi et al. (17)]. (B) MM.1S cells were cultured with tubacin $(2.5$ and $5 \mu \mathrm{M})$ for $8 \mathrm{~h}$. Whole-cell lysates were immunoprecipitated with anti-Ub Ab. Immununoprecipitates were subjected to Western blotting with anti-HDAC6 Ab. (C) MM.1S cells were cultured with tubacin $(2.5$ and $5 \mu \mathrm{M})$ for $8 \mathrm{~h}$. Whole-cell lysates were immunoprecipitated with anti-dynein Ab. Immununoprecipitates were then subjected to Western blotting with anti-HDAC6 and dynein Abs. (D) MM.1S and RPMI8226 cells were cultured with tubacin $(2.5$ and $5 \mu \mathrm{M})$ for $24 \mathrm{~h}$. Whole-cell lysates were subjected to Western blot with anti-Ub Ab. (E) MM.1S and RPMI8226 cells were cultured with tubacin (T: $5 \mu \mathrm{M})$ and/or bortezomib (B: $5 \mathrm{nM})$ for $12 \mathrm{~h}$. Whole-cell lysates were subjected to Western blotting with anti-Ub Ab.

tubacin $(5 \mu \mathrm{M})$ induces peak acetylation of $\alpha$-tubulin in RPMI8226 cells at $12 \mathrm{~h}$ (Fig. 1C). Importantly, expression of HDAC6 is not altered by tubacin treatment (data not shown).

Inhibitors of HDACs represent a promising treatment strategy (25). In our recent studies, we have demonstrated that both second generation of a hybrid polar compound suberoylanilide hydroxamic acid (SAHA) (26) and novel hydroxamic acid derivative NVP-LAQ824 (27) mediate anti-MM activity. Because these agents nonselectively inhibit HDACs, we also examined the effect of SAHA on acetylation of lysine in MM.1S and RPMI8226 cells. In contrast to tubacin, which specifically induces acetylation of $\alpha$-tubulin (Fig. $1 B$ and $C$ ), SAHA triggers more potent acetylation of lysine on histone $\mathrm{H} 3$ and $\mathrm{H} 4$ than $\alpha$-tubulin (Fig. 1D). These results demonstrate that HDAC6 is constitutively expressed in MM cell lines and tubacin specifically induces acetylation of $\alpha$-tubulin, confirming the specific inhibitory effect of tubacin on HDAC6 activity in MM cells.

Tubacin Inhibits MM Cell Growth. Based on the specific inhibitory effect of tubacin on HDAC6, we next examined the cytotoxicity of tubacin against drug-sensitive (MM.1S, U266, INA-6, and RPMI8226) and drug-resistant (RPMI-LR5 and RPMI-Dox40) $\mathrm{MM}$ cell lines. These cells were treated with tubacin $(1.25-20$ 
A
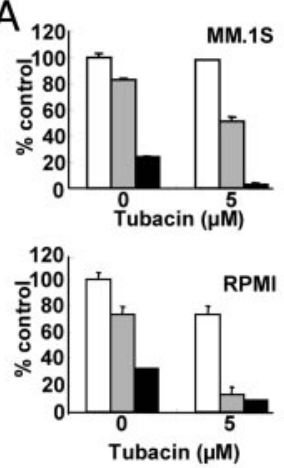

B o

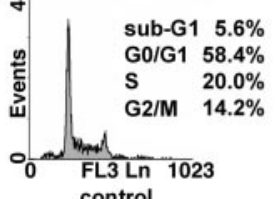

ㅇ sub-G1 $7.8 \%$

G0/G1 $37.2 \%$

\begin{tabular}{l|ll} 
& G0/G1 & $37.2 \%$ \\
& $\mathrm{~S}$ & $15.5 \%$
\end{tabular}

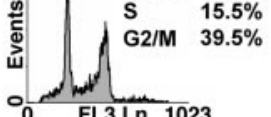

Bortezomib

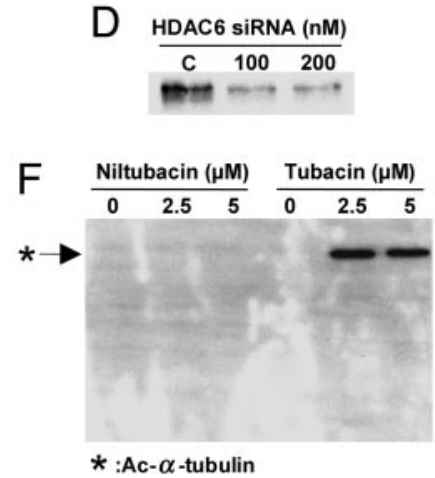

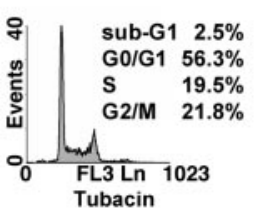

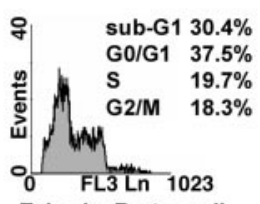

Tubacin+Bortezomib
C

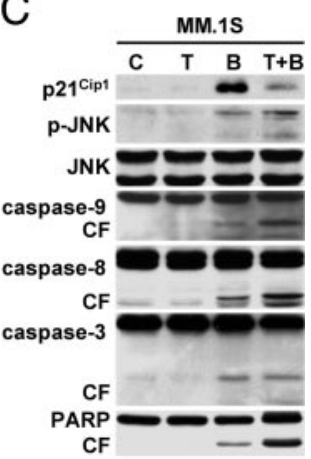

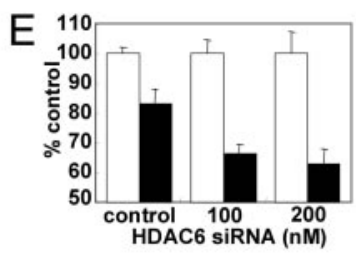

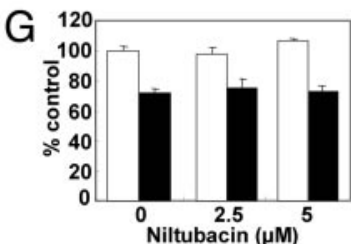

Fig. 4. Tubacin and bortezomib induce synergistic antitumor activity in MM cell lines. (A) MM.1S and RPMI8226 MM cells were cultured for $24 \mathrm{~h}$ in the presence or absence of tubacin ( $5 \mu \mathrm{M})$ in control media (white bars) and with $5 \mathrm{nM}$ (gray bars) or $10 \mathrm{nM}$ (black bars) bortezomib; cytotoxicity was assessed by MTT assay. $(B)$ MM.1S cells were cultured for $24 \mathrm{~h}$ in the presence or absence of tubacin $(5 \mu \mathrm{M})$ and/or bortezomib (5 nM); cell cycle profile was assessed by flow cytometry

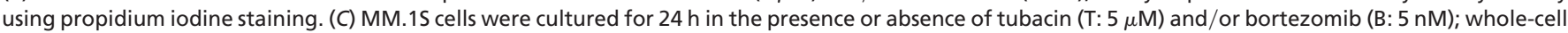

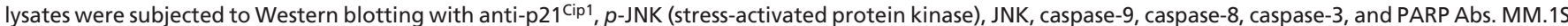

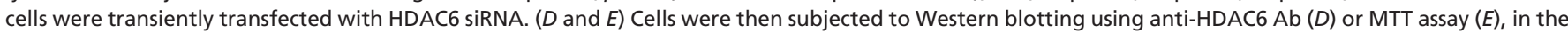
presence or absence of $5 \mathrm{nM}$ bortezomib (filled bars). MM.1S cells were cultured for $24 \mathrm{~h}$ with niltubacin $(2.5$ and $5 \mu \mathrm{M})$ or tubacin ( 2.5 and $5 \mu \mathrm{M})$. ( $F$ and $G)$ Cells were then subjected to Western blotting using Ac-Lys Ab $(F)$ or MTT assay $(G)$, in the presence or absence of $5 \mathrm{nM}$ bortezomib (filled bars). Data represent mean $( \pm S D)$ of quadruplicate cultures.

$\mu \mathrm{M}$ ) for 48 (Fig. $2 A$ ) and $72 \mathrm{~h}$ (Fig. $2 B$ ), and cytotoxicity was assessed by MTT assay as described (11). Tubacin significantly inhibits both drug-sensitive and drug-resistant MM cell growth, with $\mathrm{IC}_{50} 5-20 \mu \mathrm{M}$ at $72 \mathrm{~h}$. The most sensitive and resistant cell lines are RPMI8226 and MM.1R cells, respectively (Fig. 2B). Importantly, no cytotoxicity in peripheral blood mononuclear cells (PBMCs) is induced by tubacin (Fig. 2C). These results indicate that tubacin sensitivity is independent of resistance to conventional chemotherapeutic agents (dexamethasone, melphalan, and doxorubicin) and suggest a favorable therapeutic index in tumor cells versus normal cells. Because we have shown that HDAC inhibitors SAHA and NVP-LAQ824 trigger MM cell death by caspase-dependent apoptosis, we studied whether tubacin-induced cytotoxicity is also mediated by apoptosis. In MM.1S and RPMI8226 cells treated with tubacin $(10 \mu \mathrm{M})$ for 0-24 h, time-dependent caspase-8/PARP cleavage is induced (Fig. 2D). We further confirmed apoptotic cell death in tubacintreated MM cells by using APO2.7 staining (Fig. 2E). APO2.7positive cells increased from $8.1 \%$ to $30.3 \%$ and $41 \%$ after 48 and $72 \mathrm{~h}$ of treatment with tubacin, respectively. These data indicate that tubacin-induced cytotoxicity in MM cells is mediated by caspase-dependent apoptosis.

Tubacin Inhibits Interaction of HDAC6 with Dynein; When Combined with Bortezomib, It Induces Accumulation of Ubiquitinated Proteins. Recent studies have demonstrated that polyubiquitinated proteins are degraded via both proteasome and aggresome pathways
(17); we therefore hypothesized that combined treatment directed against HDAC6 and proteasome activities that used tubacin and bortezomib, respectively, could trigger significant accumulation of polyubiquitinated proteins, followed by cell stress and cytotoxicity (Fig. $3 A$ ).

HDAC6 constitutively binds both polyubiquitinated misfolded proteins and dynein, thereby recruiting misfolded protein cargo to dynein motors for transport to aggresomes along microtubules (17). We therefore first examined whether inhibition of HDAC6 activity by tubacin alters the interaction of HDAC6 with Ub and/or dynein. HDAC6 is consistently coimmunoprecipitated with polyubiquitinated proteins (Fig. $3 B$ ) and dynein (Fig. $3 C$ ) in MM.1S cells. After treatment with tubacin $(2.5$ and $5 \mu \mathrm{M}$ for $8 \mathrm{~h}$ ), coimmunoprecipitation of HDAC6 with dynein is markedly inhibited in a dose-dependent fashion, whereas coimmunoprecipitation between HDAC6 and ubiquitinated proteins is unaffected (Fig. 3B). Importantly, HDAC1 is not coimmunoprecipitated with polyubiquitinated proteins (data not shown), suggesting specificity of interaction of HDAC6 and polyubiquitinated proteins. We next examined the impact of tubacin on polyubiquitination of proteins. As expected, polyubiquitinated proteins significantly accumulate in tubacin-treated RPMI8226 cells; however, no significant change was recognized in treated MM.1S cells (Fig. 3D), suggesting compensatory proteasomal degradation of polyubiquitinated proteins. These results also indicate that degradation of polyubiquitinated protein in RPMI8226 cells depends more on aggresomes than proteasomes, 
A

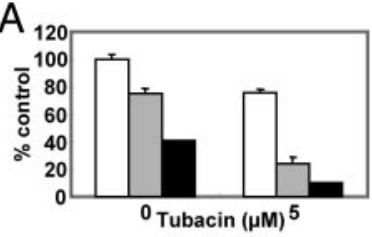

B

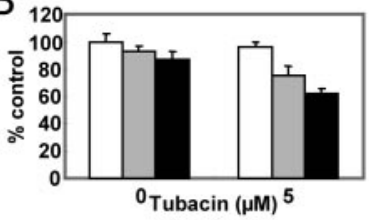

C

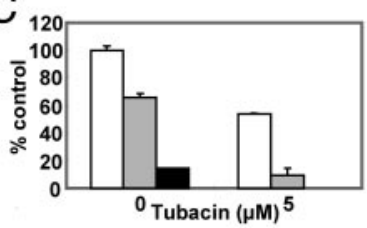

D
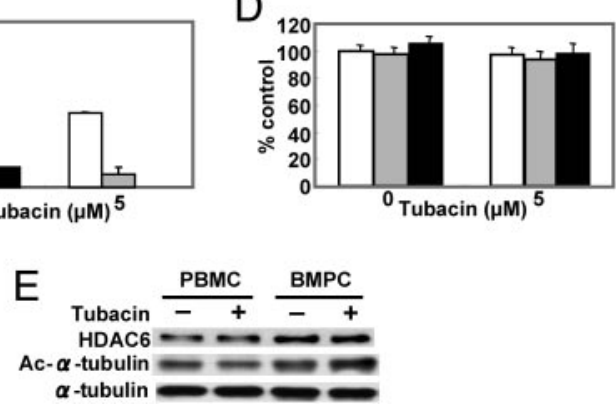

Fig. 5. Tubacin synergistically enhances bortezomib-induced cytotoxicity in patient MM cells without cytotoxicity to PBMCs. $(A-D)$ BMPCs $(A-C)$ and PBMCs $(D)$ from three MM patients were cultured in the presence or absence of tubacin $(5 \mu \mathrm{M})$ in control media (white bars) and with 10 (gray bars) or 20 $\mathrm{nM}$ (black bars) bortezomib for $24 \mathrm{~h}$; cytotoxicity was assessed by MTT assay. (E) MM patient PBMCs were cultured in the presence or absence of tubacin (5 $\mu \mathrm{M})$. Whole-cell lysates were subjected to Western blotting with anti-HDAC6, Ac-Lys, or $\alpha$-tubulin Abs.

consistent with MTT data demonstrating that RPMI8228 cells are more sensitive to tubacin than MM.1S cells (Fig. $2 A$ and $B$ ). Importantly, combined tubacin $(5 \mu \mathrm{M})$ and bortezomib $(5 \mathrm{nM})$ dramatically augment accumulation of polyubiquitinated proteins in both MM.1S and RPMI8226 cells, compared with either agent alone (Fig. 3E). These results further indicate that degradation of polyubiquitinated occurs in both proteasomes and aggresomes; therefore, inhibiting both pathways induces significant accumulation of polyubiquitinated proteins in MM cells.

Synergistic Anti-MM Activity of Tubacin with Bortezomib Is Mediated by JNK-Caspase Activation. Having shown significant accumulation of polyubiquitinated proteins after combined treatment with tubacin and bortezomib, we next examined whether combination treatment could also induce significant cytotoxicity in MM cells. As expected, tubacin synergistically enhances bortezomibinduced cytotoxicity in both MM.1S and RPMI8226 cells. For example, 5 and $10 \mathrm{nM}$ bortezomib trigger $26 \%$ and $66 \%$ RPMI8226 cell death, respectively, which is increased to $87 \%$ (combination index 0.334) and 91\% (combination index 0.370), respectively, when combined with $5 \mu \mathrm{M}$ tubacin (Fig. 4A). Similar synergistic cytotoxicity is observed in MM.1S cells (Fig. $4 A$ ). To analyze the mechanism whereby this combination treatment mediates synergistic anti-MM toxicity, we next performed cell cycle profiling in MM.1S cells. Tubacin $(5 \mu \mathrm{M})$ alone does not alter cell cycle profile, whereas bortezomib $(5 \mathrm{nM})$ alone triggers increased (14.2-39.5\%) $\mathrm{G}_{2} \mathrm{M}$ phase MM.1S cells, as in our previous studies (4); importantly, the combination of tubacin and bortezomib triggers significantly increased (5.6$30.4 \%$ ) sub- $\mathrm{G}_{0} / \mathrm{G}_{1}$ phase cells, suggesting that combination treatment triggers apoptotic cell death (Fig. $4 B$ ). Because we have shown that bortezomib (4) and other HDAC inhibitors (26, 27 ) induce $\mathrm{p} 21^{\mathrm{Cip} 1}$ in MM.1S cells, we further examined expression of $\mathrm{p} 21^{\mathrm{Cip} 1}$. Consistent to cell cycle profile, tubacin does not trigger induction of $\mathrm{p} 21^{\mathrm{Cip} 1}$. Importantly, tubacin inhibits induction of $\mathrm{p} 21^{\mathrm{Cip} 1}$ induced by bortezomib (Fig. $4 C$ ).

Because accumulation of polyubiquitinated proteins induces a cell stress response, we next examined whether this combination
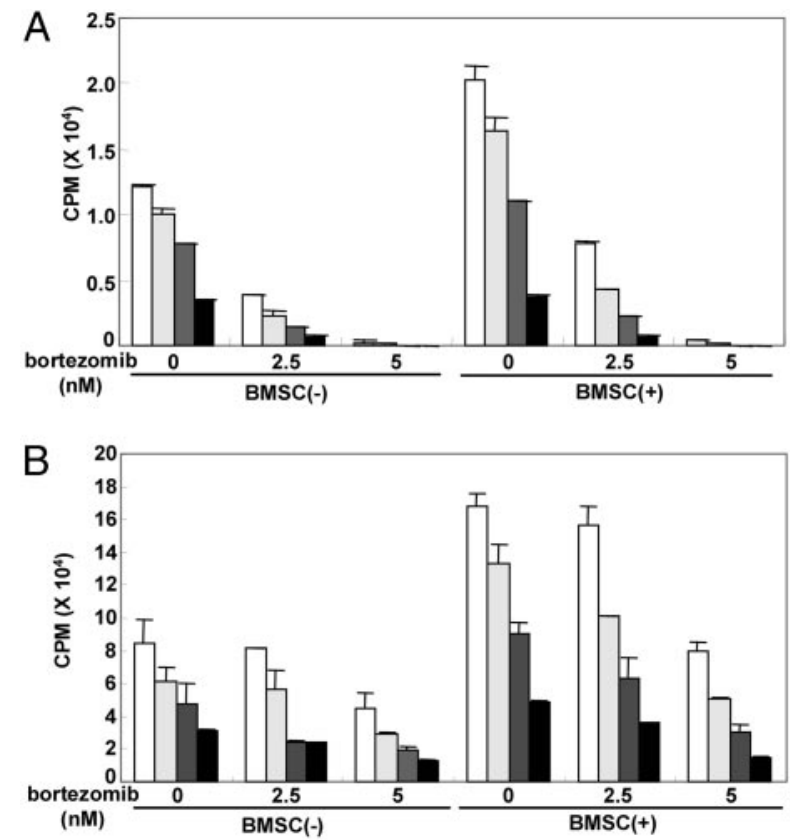

Fig. 6. Tubacin inhibits paracrine MM cell growth. MM.1S $(A)$ and RPMI8226 $(B)$ cells were cultured for $24 \mathrm{~h}$ in BMSC-coated or noncoated plates in control media (white bars) and with 1.25 (light gray bars), 2.5 (dark gray bars), or $5 \mu \mathrm{M}$ (black bars) tubacin, in the presence or absence of bortezomib ( 2.5 and $5 \mu \mathrm{M})$. DNA synthesis was assessed by $\left[{ }^{3} \mathrm{H}\right]$ thymidine uptake; data represent mean $( \pm S D)$ of quadruplicate cultures.

treatment of MM.1S cells triggers activation of JNK (also known as stress-activated protein kinase), a hallmark of cell stress response, followed by caspase cleavage, as described in our previous studies $(9,22)$. Tubacin alone does not trigger phosphorylation of JNK or caspase/PARP cleavage, and bortezomib alone induces only modest phosphorylation of JNK as well as caspase-9, caspase-8, caspase-3, and PARP cleavage (Fig. 4C). Of great interest, combined tubacin and bortezomib treatment markedly augments both JNK phosphorylation and caspase/ PARP cleavage in MM.1S cells (Fig. 4C), consistent with cytotoxicity assays (Fig. 4A). Other cell stress response-related proteins (i.e., hsp-70 and Grp78) are also induced by this combination treatment (data not shown). These results indicate that tubacin inhibits the $\mathrm{G}_{2}$ phase arrest induced by bortezomib, thereby facilitating apoptosis mediated by stress-induced JNK activation, followed by caspase/PARP cleavage.

To identify the specific role of HDAC6 inhibition in mediating synergistic MM cell cytotoxicity with bortezomib, we transiently transfected MM.1S cells with HDAC6 siRNA as described (11). HDAC6 protein expression is significantly down-regulated by transfection (Fig. 4D); importantly, bortezomib significantly increases cytotoxicity in transfectants in a dose-dependent fashion (Fig. 4E). In contrast, niltubacin, an inactive carboxylic acid tubacin analog, does not affect either acetylation of $\alpha$-tubulin (Fig. $4 F$ ) or enhance cytotoxicity in MM.1S cells induced by bortezomib (Fig. 4G). Similar results were observed in RPMI8226 cells (data not shown). These results show that inhibition of HDAC6 specifically augments bortezomib-induced cytotoxicity in MM.

Tubacin Combined with Bortezomib Demonstrates Significant Cytotoxicity in MM Patient Plasma Cells. Having show significant cytotoxicity of combined treatment of tubacin and bortezomib in MM cell lines, we further examined the effect of the combination in isolated CD138-positive MM patient BMPCs. These BMPCs 
were cultured for $24 \mathrm{~h}$ with or without tubacin $(5 \mu \mathrm{M})$, in the presence or absence of bortezomib (5 and $10 \mathrm{nM})$. Consistent with MM cell line data, cytotoxicity in BMPCs induced by bortezomib is markedly augmented by tubacin (Fig. $5 A-C$ ); importantly, no toxicity is recognized in normal PBMCs similarly treated (Fig. 5D).

We next examined the mechanism whereby combined tubacin with bortezomib specifically induces cytotoxicity in MM patient plasma cells, but not in PBMCs. Both PBMCs and BMPCs obtained from the same MM patient were treated for $12 \mathrm{~h}$ with tubacin $(5 \mu \mathrm{M})$. Constitutive expression of HDAC6 is relatively higher in BMPCs than PBMCs; importantly, acetylation of $\alpha$-tubulin is markedly enhanced by tubacin in BMPCs, but not in PBMCs (Fig. 5E).

Tubacin Combined with Bortezomib Inhibits Paracrine MM Cell Growth. We have previously shown that the BM microenvironment confers cell growth and drug resistance in MM cells $(3,28$, 29 ), and we next studied the functional sequelae of HDAC6 inhibition in the presence or absence of bortezomib, in MM cells within the BM milieu. MM.1S and RPMI8226 cells were cultured with or without BMSCs, in the presence or absence of tubacin (2.5 and $5 \mu \mathrm{M})$ and/or bortezomib (2.5-10 $\mathrm{nM})$. MM cell adherence to BMSCs triggers increased $\left[{ }^{3} \mathrm{H}\right]$ thymidine uptake of both MM.1S cells (1.75-fold, $P<0.01$ ) (Fig. $6 A$ ) and RPMI8226 cells (2.0-fold, $P<0.01$ ) (Fig. $6 B)$. Either tubacin or bortezomib

1. Gregory, W. M., Richards, M. A. \& Malpas, J. S. (1992) J. Clin. Oncol. 10, 334-342.

2. Attal, M., Harousseau, J. L., Facon, T., Guilhot, F., Doyen, C., Fuzibet, J. G., Monconduit, M., Hulin, C., Caillot, D., Bouabdallah, R., et al. (2003) N. Engl. J. Med 349, 2495-2502.

3. Hideshima, T. \& Anderson, K. C. (2002) Nat. Rev. Cancer 2, 927-937.

4. Hideshima, T., Richardson, P., Chauhan, D., Palombella, V., Elliott, P., Adams, J. \& Anderson, K. C. (2001) Cancer Res. 61, 3071-3076.

5. Mitsiades, N., Mitsiades, C. S., Poulaki, V., Chauhan, D., Gu, X., Bailey, C., Joseph, M., Libermann, T. A., Treon, S. P., Munshi, N. C., et al. (2002) Proc. Natl. Acad. Sci. USA 99, 14374-14379.

6. Hideshima, T., Chauhan, D., Richardson, P., Mitsiades, C., Mitsiades, N., Hayashi, T., Munshi, N., Dang, L., Castro, A., Palombella, V., et al. (2002) J. Biol. Chem. 277, 16639-16647.

7. Mitsiades, N., Mitsiades, C. S., Richardson, P. G., Poulaki, V., Tai, Y. T., Chauhan, D., Fanourakis, G., Gu, X., Bailey, C., Joseph, M., et al. (2003) Blood 101, 2377-2380.

8. Chauhan, D., Li, G., Shringarpure, R., Podar, K., Ohtake, Y., Hideshima, T. \& Anderson, K. C. (2003) Cancer Res. 63, 6174-6177.

9. Hideshima, T., Mitsiades, C., Akiyama, M., Hayashi, T., Chauhan, D., Richardson, P., Schlossman, R., Podar, K., Munshi, N. C., Mitsiades, N. \& Anderson, K. C. (2003) Blood 101, 1530-1534.

10. Hideshima, T., Chauhan, D., Hayashi, T., Akiyama, M., Mitsiades, N., Mitsiades, C., Podar, K., Munshi, N. C., Richardson, P. G. \& Anderson, K. C. (2003) Oncogene 22, 8386-8393.

11. Hideshima, T., Podar, K., Chauhan, D., Ishitsuka, K., Mitsiades, C., Tai, Y.-Z., Hamasaki, M., Raje, N., Hideshima, H., Schreiner, G., et al. (2004) Oncogene 23, 8766-8776.

12. Hideshima, T., Chauhan, D., Schlossman, R. L., Richardson, P. R. \& Anderson, K. C. (2001) Oncogene 20, 4519-4527.

13. Richardson, P. G., Barlogie, B., Berenson, J., Singhal, S., Jagannath, S., Irwin, D., Rajkumar, S. V., Srkalovic, G., Alsina, M., Alexanian, R., et al. (2003) N. Engl. J. Med. 348, 2609-2617.

14. Kopito, R. R. (2000) Trends Cell Biol. 10, 524-530. alone inhibits BMSC-induced $\left[{ }^{3} \mathrm{H}\right]$ thymidine uptake in a dosedependent fashion $(P<0.01)$. Importantly, tubacin significantly enhances bortezomib-induced inhibition of $\left[{ }^{3} \mathrm{H}\right]$ thymidine uptake in adherent MM.1S (Fig. 6A) and RPMI8226 (Fig. 6B) cells. The viability of BMSCs, assessed by MTT assay, is not altered by combination treatment (data not shown). These data indicate that combined treatment of tubacin with bortezomib triggers synergistic selective antitumor activity against MM cells in the $\mathrm{BM}$ milieu, thereby overcoming cell adhesion-mediated resistance to conventional therapies. In conclusion, these results strongly suggest that dual inhibition of the aggresome and proteasome with tubacin and bortezomib, respectively, synergistically enhances MM cytotoxicity. They provide the framework for clinical trials designed to enhance sensitivity and overcome resistance to bortezomib, thereby improving patient outcome in MM.

Tubacin was discovered at the small-molecule screening centered sponsored by the National Cancer Institute's Initiative for Chemical Genetics. This study was supported by National Institutes of Health Grants SPORE IP50 CA10070-01, PO-1 78378, and RO-1 CA 50947; the Doris Duke Distinguished Clinical Research Scientist Award (to K.C.A.); the Multiple Myeloma Research Foundation (T.H. and J.E.B.); the Cure for Myeloma Research Fund (K.C.A.); National Institutes of Health Grant T32-HL07623-18 (to J.E.B.); and National Institutes of Health Grant NIGMS 38627 (to S.L.S.). S.L.S. is an Investigator at the Howard Hughes Medical Institute Laboratory located in the Department of Chemistry and Chemical Biology, Harvard University.

15. Bennett, E. J., Bence, N. F., Jayakumar, R. \& Kopito, R. R. (2005) Mol. Cell 17, 351-365.

16. Garcia-Mata, R., Gao, Y. S. \& Sztul, E. (2002) Traffic 3, 388-396.

17. Kawaguchi, Y., Kovacs, J. J., McLaurin, A., Vance, J. M., Ito, A. \& Yao, T. P. (2003) Cell 115, 727-738.

18. Haggarty, S. J., Koeller, K. M., Wong, J. C., Grozinger, C. M. \& Schreiber, S. L. (2003) Proc. Natl. Acad. Sci. USA 100, 4389-4394.

19. Haggarty, S. J., Koeller, K. M., Wong, J. C., Butcher, R. A. \& Schreiber, S. L. (2003) Chem. Biol. 10, 383-396.

20. Wong, J. C., Hong, R. \& Schreiber, S. L. (2003) J. Am. Chem. Soc. 125, 5586-5587.

21. Uchiyama, H., Barut, B. A., Mohrbacher, A. F., Chauhan, D. \& Anderson, K. C (1993) Blood 82, 3712-3720.

22. Hideshima, T., Chauhan, D., Hayashi, T., Podar, K., Akiyama, M., Mitsiades, C., Mitsiades, N., Gong, B., Bonham, L., de Vries, P., et al. (2003) Cancer Res. 63, 8428-8436.

23. Le Gouill, S., Podar, K., Amiot, M., Hideshima, T., Chauhan, D., Itshitsuka, K., Kumar, S., Raje, N., Richardson, P. G., Harousseau, J. L. \& Anderson, K. C. (2004) Blood 104, 2886-2892.

24. Raje, N., Kumar, S., Hideshima, T., Ishitsuka, K., Chauhan, D., Mitsiades, C., Podar, K., Le Gouill, S., Richardson, P., Munshi, N. C., et al. (2004) Blood 104, 4188-4193.

25. Marks, P. A., Miller, T. \& Richon, V. M. (2003) Curr. Opin. Pharmacol. 3, 344-351.

26. Mitsiades, N., Mitsiades, C. S., Richardson, P. G., McMullan, C., Poulaki, V., Fanourakis, G., Schlossman, R., Chauhan, D., Munshi, N. C., Hideshima, T., et al. (2003) Blood 101, 4055-4062.

27. Catley, L., Weisberg, E., Tai, Y. T., Atadja, P., Remiszewski, S., Hideshima, T. Mitsiades, N., Shringarpure, R., LeBlanc, R., Chauhan, D., et al. (2003) Blood 102, 2615-2622.

28. Hideshima, T., Richardson, P. \& Anderson, K. C. (2003) Immunol. Rev. 194, 164-176.

29. Mitsiades, C. S., Mitsiades, N. S., McMullan, C. J., Poulaki, V., Shringarpure, R., Akiyama, M., Hideshima, T., Chauhan, D., Joseph, M., Libermann, T. A. et al. (2004) Cancer Cell 5, 221-230. 